\title{
Vascular Tissue Engineering
}

\author{
Chiara Arrigoni, Davide Camozzi, and Andrea Remuzzi
}

Department of Biomedical Engineering, Mario Negri Institute for Pharmacological Research,24125 Bergamo, Italy

\begin{abstract}
Reconstructive surgery using autologous vessels is the conventional approach for substitution of diseased vessels or for generation of bypass to improve blood supply downstream of stenosed vessels. In some circumstances the use of autologous material is not possible due to concomitant diseases or previous use, and artificial grafts must be used. Unfortunately, these grafts cannot substitute small-caliber arterial vessels because of thrombotic complications. The objective of tissue engineering at the vascular level is then to generate biological substitutes of arterial conduits with functional characteristics of native vessels, combining cellular components with biodegradable scaffolds. These research projects started in several laboratories, in the late 1990s, and have expanded in different directions using a number of experimental approaches. The objective of this review is to give an overview of the results so far obtained in this area of research, and to discuss the problems related to these investigations, at the experimental and clinical level. The article provides an overview of different biodegradable scaffolds used, experimental techniques for vessels maturation in vitro under mechanical stimulation, and of differentiated as well as precursors of vascular cells, which opens new opportunities for further development of this form of cell transplantation. Finally, the current available results in clinical research will be discussed.
\end{abstract}

Key words: Vascular tissue engineering; Scaffolds; Bioreactors; Vascular cells

\section{INTRODUCTION}

Cardiovascular diseases (CVD) are the leading cause of mortality in Western countries. In the US $38 \%$ of all deaths are related to CVD (2). Conventional therapeutic strategies of CVD include angioplasty, with or without stent application, and endoarterectomy or bypass grafting when the occlusion degree of the vessel is more severe (11). The graft usually consists of either artery or vein segments harvested from the patient. The supply of healthy native vessels, however, is limited by the preexistence of pathological conditions or by previous surgical intervention. Moreover, the use of a venous tract in the arterial circulation often results in a process of vessel remodeling, with neointimal thickening that can lead to vessel stenosis or aneurysm formation (1). Artificial vascular grafts (such as Dacron fabric or expanded polytetrafluoroethylene) are now conventionally used for large arteries but are not suitable for small-caliber vessels, smaller than $6 \mathrm{~mm}$ in diameter (15).The occlusion of these grafts may be caused by thrombus formation or, at longer time periods, by intimal hyperplasia, particularly near anastomosis sites, that rapidly leads to graft occlusion (10). To overcome this limitation, several research groups focused in the last 10 years on the generation of a biological vascular substitute using tissue engineering techniques. These grafts must in principle be infection resistant, biocompatible, and nonthrombogenic. In addition, they must have mechanical properties matching those of native vessels with the ability to respond to physiological stimuli, with vasoactive response, and structure remodeling consequent to environmental modifications (Table 1).

Tissue engineering (TE) is a multidisciplinary approach to provide living tissue products to restore, maintain, or improve tissue functions (17). For generation of connective tissue substitutes in vitro, cells are seeded onto a biodegradable material (scaffold) and the construct is developed with biochemical and biomechanical stimulation, to enable cells to remodel the scaffold and to produce extracellular matrix (ECM).

Pioneering work on vascular tissue engineering started in the late 1980s with the study published by Weinberg and Bell (37), but the first remarkable results were those of L'Hereux and coworkers in 1998 (18). In their work, a tissue engineered blood vessel (TEBV) was produced, with human cells and without a support material. Human umbilical vein, smooth muscle cells (SMC), and skin fibroblasts were grown in culture in the presence of sodium ascorbate, to induce ECM formation.

Address correspondence to Andrea Remuzzi, Eng.D., Department of Biomedical Engineering, Mario Negri Institute for Pharmacological Research, Via Gavazzeni, 11, 24125 Bergamo, Italy. Tel: +39 (035) 319.888; Fax: +39 (035) 319.331; E-mail: aremuzzi@ marionegri.it 
Table 1. Structural and Functional Properties of Arterial Grafts

\begin{tabular}{lccc}
\hline & $\begin{array}{c}\text { Synthetic } \\
\text { Grafts }\end{array}$ & $\begin{array}{c}\text { Autologous } \\
\text { Vessels } \\
\text { Tract }\end{array}$ & $\begin{array}{c}\text { Tissue } \\
\text { Engineered } \\
\text { Grafts }\end{array}$ \\
\hline Long-term patency & no & yes & yes \\
Nonthrombogenicity & no & yes & yes \\
Resistant to infections & no & yes & yes \\
Ability to remodel & no & no & yes \\
Absence of immunologic reaction & yes & yes & yes \\
Compliance & no & yes & yes \\
Ability to grow & no & no & yes \\
Sufficient burst strength & yes & yes & yes \\
Ease of handling & yes & yes & yes \\
Uncomplicated storage & yes & no & no \\
"Off the shelf" availability & yes & no & no \\
\hline
\end{tabular}

After 30 days cells formed cohesive sheets that were removed from culture flasks. The SMC sheet was wrapped around a mandrel to produce the media of the vessel; after a week of maturation, the fibroblast sheet was rolled around the media to provide the adventitia layer and then the construct was left in culture for 7 weeks. The mechanical properties of the TEBV were higher than that of human saphenous vein, with morphology of wall organization comparable to that of native arteries. This layered vascular construct was implanted in dogs, showing good suturability and no sings of degradation or dilatation after 7 days of implantation. On the other hand, intramural blood infiltration was observed between vessel layers. Long-term patency could not be verified in this study because of the lack of an endothelial lining.

In 1999 Niklason and coworkers (23) described the development of a TEBV, based on bovine SMC and endothelial cells (EC), seeded onto a polyglicolic acid (PGA) mesh. The construct formed by SMC on the scaffold was cultured for 8 weeks in a bioreactor, to expose the vessel lumen to a pulsatile pressure (165 bpm, 5\% radial distention), through a silicon sleeve. Three days before harvesting, the sleeve was removed and the lumen of the vessel was seeded with endothelial cells, then medium flow was applied directly to the cultured vessel. Histology of the TEBV showed the construct wall homogeneously colonized by SMCs, with few PGA residues. These vessels also displayed contractions in response to serotonin and prostaglandin stimulation in vitro. To assess in vivo performance, vascular constructs made of porcine SMC and EC were implanted in minipigs. Digital angiography at different time points was performed and documented patency of pulsed grafts for up to 4 weeks.

A different approach was that followed by Kaushal and coworkers (14). They used a decellularized vascular vessel prepared from porcine iliac arteries as scaffold, and seeded the material with endothelial progenitor cells (EPC). Cells were isolated from peripheral blood of a sheep using a density gradient and then grown in culture for 7 weeks, before seeding. After having obtained a confluent endothelial layer, the grafts were subjected to shear stress preconditioning, to enhance cell retention. Nitric oxide production by EPC was assessed to verify the ability of fluid perfusion through the seeded grafts to relax aorta segments previously contracted. The engineered vessels were implanted in vivo and remained patent for up to 130 days. At the time of harvest, however, some degree of intimal thickening of the grafts was observed, due to host cells infiltration.

These first results demonstrated the feasibility of these experimental approaches but they seem to be time consuming, requiring more than 8 weeks in some instance for vessel maturation. Another drawback of these techniques regards mechanical properties of the scaffold that are far from being elastic, like native vessels. Another important limitation of these studies is that the use of differentiated vascular SMC and EC cells from autologous vascular tissue requires harvesting an arterial or venous vessel segment from the patient. Present research lines focus on these aspects, trying to find a solution that can lead to improved engineered vascular prostheses. In the following we discuss the research activities in this context that are aimed at overcoming these limitations.

\section{BIODEGRADABLE SCAFFOLDS FOR VASCULAR TISSUE ENGINEERING}

Several reports in the literature describe the use of different biomaterials, either of natural origin, like decellularized matrices or collagen sponges, or synthetic products, like PGA, polycaprolactone (PCL), and other 
polymers. The main advantages related to the use of natural acellular tissue are the intrinsic cell compatibility and adequate mechanical properties, due to material composition. The presence of ECM proteins can in fact support cellular attachment and growth, and provides the desired elasticity and mechanical strength. On the other hand, natural tissues need to be treated with chemicals to neutralize cellular components and may activate host immune response with risk of patient sensibilization (28). Among natural materials, collagen matrices were also used in several studies. A recent work of Berglund and colleagues (3) reports on a TEBV based on a crosslinked collagen sleeve, used as mechanical support, surrounded by collagen hydrogel containing vascular SMCs. The presence of a biological sleeve provided temporary mechanical support while cell-mediated remodeling of the construct occurred, afterwards degrading over time. The results obtained showed that this method allowed to obtain a vessel with higher strength compared to collagen gels alone but still weaker then native arteries. Another material obtained from a biological molecule is HYAFF®, a benzilic ester of hyaluronic acid. This material, approved for clinical use, is routinely implanted for skin and cartilage reconstruction. In the field of vascular tissue engineering we recently reported (26) the possibility of generating a vascular construct starting from cellularized flat sheets made of nonwoven $\mathrm{HYAFF} \AA$ rolled over a mandrel, as shown in Figure 1. SMC showed a good affinity with the scaffold material and matrix production was detected. However, mechanical and histological properties of the constructs are still far from those of natural arteries.

Among synthetic materials, PGA is the more frequently used, together with PCL and polyhydroxybutyrate (P4HB). In 2001 Watanabe and colleagues (36) published a study reporting successful generation of a tissue engineered autograft, based on PCL seeded with myofibroblast, cultured for 1 week, and implanted in the inferior vena cava of the same dog from which cells were obtained. Tensile tests performed on the scaffold without cells demonstrated that the properties of the material degraded in only 1 week, and explanted grafts, after 6 months, showed no sign of stenosis or aneurismal change, even in the absence of an endothelial layer and without the use of anticoagulants. Another interesting study based on a synthetic biomaterial is that of Hoerstrup and coworkers (12). In this work myofibroblasts and endothelial cells isolated from segments of ovine carotid were seeded onto tubes made of a copolymer of PGA and P4HB and cultured in a bioreactor. Mechanical properties of these constructs improved with culture time in the presence of mechanical stimulation. However, despite the variety of biomaterials described in the literature, these materials do not possess the required properties, mainly elasticity that characterizes native vessels, and adequate compatibility with vascular cells. In addition, the major limitation in the development of new synthetic biodegradable materials is the approval from public agencies for clinical use. This process is time consuming and very expensive and is becoming an obstacle in development of new materials.

\section{THE USE OF BIOREACTORS}

It has been suggested by several research groups that maturation of vascular constructs must be carried out in vitro in the presence of a physical stimulation similar to that present in native arteries. To this purpose cell bioreactors have been developed to subject vascular constructs to radial dilatation or fluid flow with pulsatile pressure. One of the first examples of these devices was that described by Seliktar and coworkers (29) that was based on a pulsatile pressure generated inside a silicone sleeve surrounded by a collagen gel seeded with SMCs. Bioreactors developed by Sodian et al. (33) and by
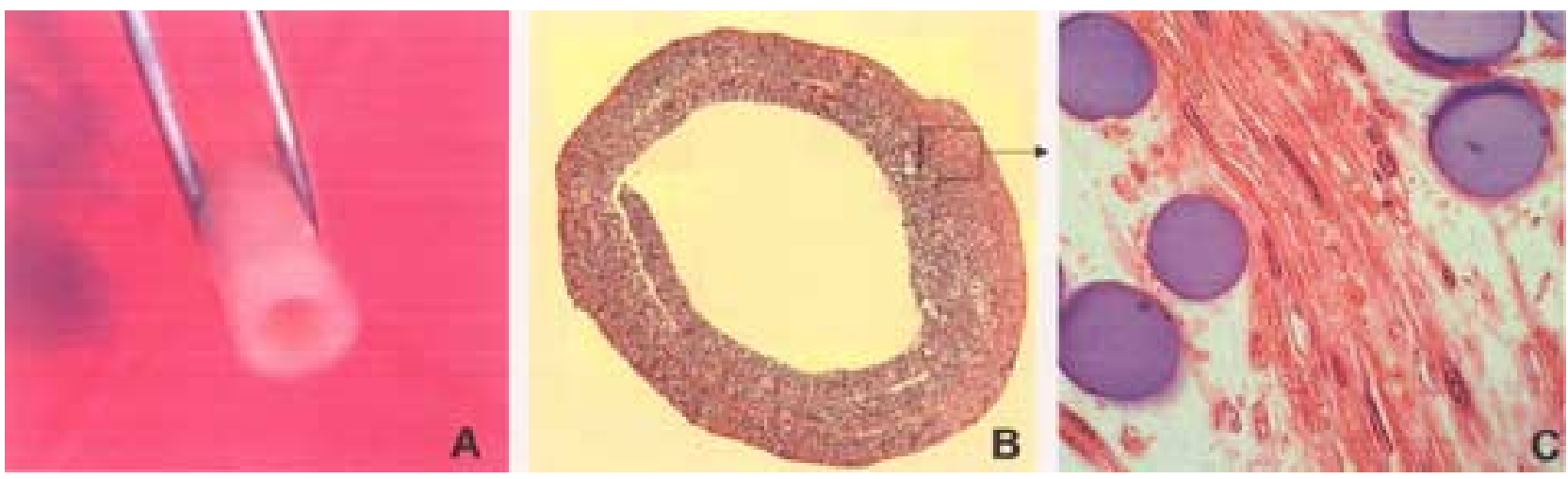

Figure 1. Representative image of $\mathrm{HYAFF}{ }^{\circledR}$ vascular constructs (A). Hematoxylin and eosin staining of construct section perpendicular to its main axis at low (B) and high (C) magnification showing cells grown within the construct wall. 
Thompson et al. (35) in 2002 were based on a different type of mechanical stimulation imposed to vascular constructs. Fluid flow was directly applied to the developing constructs, submitting them to physiological luminal shear stress and radial pressure, enhancing nutrient and oxygen delivery. The device described by Sodian and coworkers was driven by a mechanical ventilator that generated a sinusoidal pressure wave, transmitted to culture medium by a silicon membrane. Seeding of vascular cells onto the tubular scaffold was performed in the same chamber used for perfusion, thus limiting the risks of contamination. Thompson and colleagues developed a perfusion bioreactor composed of a closed circuit with an oxygenator to provide oxygen to the circulating culture medium. The pressure was generated by a mechanical ventilator and the presence of valves and compliant tubing allowed to obtain a pressure wave similar to physiological aortic waveforms. The results showed that the device successfully supported growth and maturation of vascular constructs. Recently Williams and coworkers (38) developed a perfusion bioreactor in which cell seeding and mechanical stimulation of the vascular construct were performed. Seeding of cells onto the polymer was achieved by means of a dual syringe pump that pumped the cell suspension reciprocally through the lumen of the scaffold. After cell seeding, fluid flow was applied to the vascular construct, both through the vessel lumen and in the direction perpendicular to construct longitudinal axis, to further increase nutrient and oxygen supply. Constructs developed with the bioreactor showed that cells were distributed uniformly, mainly on the luminal surface, demonstrating the efficiency of this seeding method. In constructs harvested after 25 days of culture, cells heavily colonized the external regions of construct wall, stained positively for $\alpha$-smooth muscle actin ( $\alpha$ SMA) and calponin and some amount of elastin was detected.

Another recent example of a new bioreactor was reported by McCulloch and coworkers (21). This device applies radial pressure and fluid flow to the vascular construct and stretches the vessel in the longitudinal direction. The system used to generate physiological pressure and flow waveforms is composed by a computercontrolled pulsatile pump, connected to the chamber that contains the vascular construct and to a reservoir with an inlet valve that emulates systemic resistance. The straininducing system was composed of a rack-and-pinion mechanism driven by a precision stepper motor, fastened to a tissue holder. Functional tests confirmed the possibility to obtain the desired mechanical outputs and to support constructs maturation. The group of Optiz and coworkers recently reported studies $(24,25)$ on the development of a TEBV, based on $\mathrm{P} 4 \mathrm{HB}$ scaffold and ovine SMC and EC. The maturation of the constructs was carried out within a bioreactor that applied pressure and luminal fluid flow. The values of shear stress imposed on the endothelial layer matched physiological range and applied pressure was variable between normal systolic and diastolic values. Mechanical stimulation allowed to obtain stronger constructs with higher ECM content compared with respective controls. The comparison with native arteries showed that bioartificial vessels had comparable strength but significantly lower elasticity.

Looking at these results, it can be observed that all the developed devices are able to mimic the in vivo conditions, in terms of shear stress and pressure applied. It remains to be further investigated which conditions of mechanical stimulation can lead to engineered constructs with properties equivalent to that of native vessel.

\section{CELLULAR SOURCES FOR VASCULAR TISSUE ENGINEERING}

Regarding cellular sources for vascular tissue engineering, the most recent studies investigated the possibility of using alternative sources to differentiated vascular cells, to avoid the need of surgical harvest of autologous vessel segments. There are two different solutions in this regard. Some investigators have focused on genetically modified SMC that can proliferate faster and for a longer time than untreated cells $(22,34)$; others are attempting to use stem cells, like bone marrow stromal cells (BMSC) $(5,16,20)$ or endothelial and smooth muscle progenitor cells $(14,39)$.

McKee and coworkers (22) hypothesized that the limiting factor that prevents in vitro engineering of a blood vessel is the limited proliferative capacity of adult SMC. They therefore developed a method to avoid SMC ageing, infecting the cells with a retroviral vector encoding for human telomerase reverse transcriptase subunit (hTERT) that has the ability to elongate cell telomeres, extending cell life span. Genetically modified cells were phenotypically normal, expressing calponin, smooth muscle heavy chain, and elastin. When seeded onto PGA mesh and cultured within a bioreactor, hTERT SMC allowed to obtain a vessel with larger wall thickness, increased strength, and decreased apoptosis, compared to control nontransfected cells. A different approach was that followed by Stegemann and colleagues (34), who attempted to avoid SMC dedifferentiation with culture passages. They investigated SMC transfection with a gene that encoded for phenotype regulating protein cyclic guanosine monophospate-dependent protein kinase (PKG). This protein is able to increase the levels of $\alpha$ SMA, driving SMC phenotype towards a more physiologic contractile phenotype. PKG transfected cells in monolayer cultures showed a higher expression of $\alpha$ SMA compared to control SMCs, but the collagen gels obtained using transfected cells were not 
significantly different from controls in terms of compactness and cellularity. Mechanical (radial pressure) and biochemical (PDGF and TGF- $\beta$ ) stimulation showed different effects on control and PKG transfected gels. In the latter the addition of PDGF did not result in lower expression of contractile proteins as in controls, demonstrating a steady differentiated phenotype of treated cells.

Regarding stem cells, there are already in the literature approaches that focuses on the use of BMSC for vascular tissue engineering. As reported in several studies, it is possible to differentiate these cells into SMCs $(8,13,27)$. The first report on BSMC to regenerate blood vessels in vivo is that of Matsumura and colleagues (20). In this study, BMSCs were extracted from canine bone marrow and treated with a fluorescent cell tracker, prior to seeding onto PCL scaffolds. A cellularized scaffold was then implanted into the same dog and immunofluorescence staining was performed at harvesting. Results demonstrated that BMSCs adhered to the scaffold and differentiated into either endothelial or smooth muscle cells, as confirmed by the presence of cells positive for specific endothelial or smooth muscle markers and simultaneously for the cell tracker. The vascular grafts remained patent for the entire implantation period and were colonized also from host cells. Moreover, generation of physiological components of the vessel wall, such as elastic laminae and circumferentially aligned collagen fibers, was observed. In 2005 Cho and coworkers (5) reported engineering of a vascular graft using predifferentiated BMSCs seeded onto decellularized vascular matrix. BMSCs were isolated from dogs, marked with a cell tracker, and differentiated in vitro in either SMC or EC, with appropriate growth factors in the culture medium. Successful cell differentiation towards a SMC phenotype was confirmed by positive staining for $\alpha \mathrm{SMA}$ and myosin heavy chain, while endothelial differentiation was demonstrated by CD31 and von Willebrand factor expression. After 1 week of in vitro culture, vascular constructs were implanted in vivo. Analysis on explanted grafts showed that the internal surface was uniformly covered with endothelial cells, derived also from the host, that ensure patency to the vascular grafts. In vitro seeded BMSCs were still present, as confirmed by fluorescence detection.

Another innovative cellular source suitable for vascular tissue engineering is represented by EPC. These cells can be isolated from peripheral blood, thus avoiding invasive surgical procedures; they can differentiate in cells with endothelial and also mesenchymal phenotype (9, 39). In the previously mentioned work by Kaushal and coworkers (14), these cells were seeded onto decellularized matrices, allowing to obtain grafts that were patent for the entire experimental period. The study reported by
Shirota and colleagues (31) described a small-diameter vascular graft obtained from EPC seeded onto a polyurethane scaffold. EPC demonstrated to be highly proliferative, to possess antithrombogenic properties, and to respond to shear stress. Recently, Simper and colleagues $(7,32)$ identified SM progenitor cells (SMPC) in peripheral blood and expanded them ex vivo. Immunofluorescence staining showed that SMPCs were positive for $\alpha$ SMA, myosin heavy chain, and calponin, and adhesion tests demonstrated their ability to adhere to fibronectincoated surface. All these findings showed that progenitor cells could potentially be used for the generation of engineered blood vessel segments.

A completely different approach regarding cell source was reported in the work of Campbell and coworkers $(4,6)$, who investigated the possibility to induce in vivo regeneration of cylindrical cell construct. Their method consisted of the insertion of a polymeric tube in the peritoneal cavity of a dog. The presence of a foreign material in the body caused the formation of a fibrotic tissue around the mandrel. This tissue was then harvested and characterized. The amount of tissue formation depended on the type of synthetic material used, and cells included in the tissue were positive for early differentiation SMC markers ( $\beta$-actin, vimentin) but not for more specific markers (myosin and elastin). Burst strength of the constructs was higher than $2500 \mathrm{mmHg}$ and, when transplanted in dogs, the grafts showed no sign of aneurysm nor intimal thickening. Three months after implantation the formation of a physiological layered structure was observed in the harvested graft, with smooth muscle cells in the middle and a luminal endothelial layer. All these studies demonstrate that next generation of bioartificial vessels may effectively be obtained without the use of differentiated vascular cells.

\section{CLINICAL APPLICATIONS OF BIOARTIFICIAL VESSELS}

Despite numerous successful studies at the experimental level, the transfer of these findings to the clinic has not yet started. There are only a few reports in the literature of successful use of tissue engineering in vascular surgery. The first clinical application of a biodegradable scaffold seeded with cells was reported by Shin'oka and colleagues in 2001 (30). Cells isolated from a peripheral vein were seeded onto a PCL-PLLA scaffold and implanted in a 4-year-old girl, to substitute a tract of pulmonary artery. Seven months after intervention the patient had no complications and the graft was still patent and without aneurysm. In a second report by the same group (19), they reported the application of a tissue engineered autograft based on either SMC from saphenous vein or BMSC from bone marrow seeded onto the same polymeric scaffold. In the first procedure 
developed, SMCs were harvested, expanded in vitro, seeded onto the scaffold, and implanted in pulmonary artery after 1 week of culture. In the second method BMSC were isolated and directly seeded onto the scaffold, which was left in culture for $2-4 \mathrm{~h}$ before implantation in the inferior vena cava. Twenty patients with different cardiovascular congenital defects, in pulmonary artery or venous sites, underwent surgical reconstruction with the engineered autografts, and clinical follow-up of four patients was reported. In all cases there were no postoperative complications and all the grafts presented adequate flow, without signs of stenosis or dilatation. Although these results were successful, it has to be noted that all implantations were performed on low-pressure sites of the circulation. Whether similar results can also be obtained for arterial segments has yet to be established.

\section{CONCLUSIONS}

In conclusion, much work remains to be done to obtain a clinically suitable engineered vascular graft. New progress is required to obtain an adequate support material that possesses elasticity and mechanical strength comparable to that of native vessels, combined with the necessary degradation rate and cellular compatibility. In addition, many issues addressing the influence of mechanical and biochemical stimuli on cellular behavior are still to be elucidated to optimize in vitro culture conditions. Finally, mechanisms underlying differentiation towards vascular phenotype of stem cells (either mesenchymal or circulating progenitor cells) must be further investigated, to use these cells as an effective source for vascular tissue engineering. All these advancements in the different research areas seem to be reachable in a midterm future and they will hopefully open new interesting clinical applications.

\section{REFERENCES}

1. Angelini, G. D.; Jeremy, J. Y. Towards the treatment of saphenous vein bypass graft failure-a perspective of the Bristol Heart Institute. Biorheology 39:491-499; 2002.

2. American Heart Association. Heart disease and stroke statistics-2005 update. www.americanheart.org.

3. Berglund, J. D.; Mohseni, M. M.; Nerem, R. M.; Sambanis, A. A biological hybrid model for collagen-based tissue engineered vascular constructs. Biomaterials 24: 1241-1254; 2003.

4. Campbell, J. H.; Efendy, J. L.; Campbell, G. R. Novel vascular graft grown within recipient's own peritoneal cavity. Circ. Res. 85:1173-1178; 1999.

5. Cho, S. W.; Lim, S. H.; Kim, K. K.; Hong, Y. S.; Kim, S. S.; Yoo, K. J.; Park, H. Y.; Jang, Y.; Chang, B. C.; Choi, C. Y.; Hwang, K. C.; Kim, B. S. Small-diameter blood vessels engineered with bone marrow-derived cells. Ann. Surg. 241:506-515; 2005.

6. Chue, W. L.; Campbell, G. R.; Caplice, N.; Muhammed, A.; Berry, C. L.; Thomas, A. C.; Bennett, M. B.; Camp- bell, J. H. Dog peritoneal and pleural cavities as bioreactors to grow autologous vascular grafts. J. Vasc. Surg. 39: 859-867; 2004.

7. Deb, A.; Skelding, K. A.; Wang, S.; Reeder, M.; Simper, D.; Caplice, N. M. Integrin profile and in vivo homing of human smooth muscle progenitor cells. Circulation 110: 2673-2677; 2004.

8. Dennis, J. E.; Charbord, P. Origin and differentiation of human and murine stroma. Stem Cells 20:205-214; 2002.

9. Dvorin, E. L.; Wylie-Sears, J.; Kaushal, S.; Martin, D. P.; Bischoff, J. Quantitative evaluation of endothelial progenitors and cardiac valve endothelial cells: Proliferation and differentiation on poly-glycolic acid/poly-4-hydroxybutyrate scaffold in response to vascular endothelial growth factor and transforming growth factor beta1. Tissue Eng. 9:487-493; 2003.

10. Greenwald, S. E.; Berry, C. L. Improving vascular grafts: The importance of mechanical and haemodynamic properties. J. Pathol. 190:292-299; 2000.

11. Hill, R.; Bagust, A.; Bakhai, A.; Dickson, R.; Dundar, Y.; Haycox, A.; Mujica Mota, R.; Reaney, A.; Roberts, D.; Williamson, P.; Walley, T. Coronary artery stents: A rapid systematic review and economic evaluation. Health Technol. Assess. 8(iii-iv):1-242; 2004.

12. Hoerstrup, S. P.; Zund, G.; Sodian, R.; Schnell, A. M.; Grunenfelder, J.; Turina, M. I. Tissue engineering of small caliber vascular grafts. Eur. J. Cardiothorac. Surg. 20: 164-169; 2001.

13. Kadner, A.; Hoerstrup, S. P.; Zund, G.; Eid, K.; Maurus, C.; Melnitchouk, S.; Grunenfelder, J.; Turina, M. I. A new source for cardiovascular tissue engineering: Human bone marrow stromal cells. Eur. J. Cardiothorac. Surg. 21: 1055-1060; 2002.

14. Kaushal, S.; Amiel, G. E.; Guleserian, K. J.; Shapira, O. M.; Perry, T.; Sutherland, F. W.; Rabkin, E.; Moran, A. M.; Schoen, F. J.; Atala, A.; Soker, S.; Bischoff, J.; Mayer, Jr., J. E. Functional small-diameter neovessels created using endothelial progenitor cells expanded ex vivo. Nat. Med. 7:1035-1040; 2001.

15. Klinkert, P.; Post, P. N.; Breslau, J. P.; van Bockel, J. H. Saphenous vein versus PTFE for above-knee femoropopliteal bypass. A review of the literature. Eur. J. Vasc. Endovasc. Surg. 27:357-362; 2004.

16. Koike, N.; Fukumura, D.; Gralla, O.; Au, P.; Schechner, J. S.; Jain, R. K. Tissue engineering: Creation of longlasting blood vessels. Nature 428:138-139; 2004.

17. Langer, R.; Vacanti, J. P. Tissue engineering. Science 260:920-926; 1993.

18. L'Heureux, N.; Paquet, S.; Labbe, R.; Germain, L.; Auger, F. A. A completely biological tissue-engineered human blood vessel. FASEB J. 12:47-56; 1998.

19. Matsumura, G.; Hibino, N.; Ikada, Y.; Kurosawa, H.; Shin'oka, T. Successful application of tissue engineered vascular autografts: Clinical experience. Biomaterials 24: 2303-2308; 2003.

20. Matsumura, G.; Miyagawa-Tomita, S.; Shin'oka, T.; Ikada, Y.; Kurosawa, H. First evidence that bone marrow cells contribute to the construction of tissue-engineered vascular autografts in vivo. Circulation 108:1729-1731; 2003.

21. McCulloch, A. D.; Harris, A. B.; Sarraf, C. E.; Eastwood, M. New multi-cue bioreactor for tissue engineering of tubular cardiovascular samples under physiological conditions. Tissue Eng. 10:565-573; 2004. 
22. McKee, J. A.; Banik, S. S.; Boyer, M. J.; Hamad, N. M.; Lawson, J. H.; Niklason, L. E.; Counter, C. M. Human arteries engineered in vitro. EMBO Rep. 4:633-638; 2003.

23. Niklason, L. E.; Gao, J.; Abbott, W. M.; Hirschi, K. K.; Houser, S.; Marini, R.; Langer, R. Functional arteries grown in vitro. Science 284:489-493; 1999.

24. Opitz, F.; Schenke-Layland, K.; Cohnert, T. U.; Starcher, B.; Halbhuber, K. J.; Martin, D. P.; Stock, U. A. Tissue engineering of aortic tissue: Dire consequence of suboptimal elastic fiber synthesis in vivo. Cardiovasc. Res. 63: 719-730; 2004.

25. Opitz, F.; Schenke-Layland, K.; Richter, W.; Martin, D. P.; Degenkolbe, I.; Wahlers, T.; Stock, U. A. Tissue engineering of ovine aortic blood vessel substitutes using applied shear stress and enzymatically derived vascular smooth muscle cells. Ann. Biomed. Eng. 32:212-222; 2004.

26. Remuzzi, A.; Mantero, S.; Colombo, M.; Morigi, M.; Binda, E.; Camozzi, D.; Imberti, B. Vascular smooth muscle cells on hyaluronic acid: Culture and mechanical characterization of an engineered vascular construct. Tissue Eng. 10:699-710; 2004.

27. Remy-Martin, J. P.; Marandin, A.; Challier, B.; Bernard, G.; Deschaseaux, M.; Herve, P.; Wei, Y.; Tsuji, T.; Auerbach, R.; Dennis, J. E.; Moore, K. A.; Greenberger, J. S.; Charbord, P. Vascular smooth muscle differentiation of murine stroma: a sequential model. Exp. Hematol. 27: 1782-1795; 1999.

28. Schmidt, C.; Baier, J. Acellular vascular tissues: Natural biomaterials for tissue repair and tissue engineering. Biomaterials $21: 2215-2231 ; 2000$.

29. Seliktar, D.; Black, R. A.; Vito, R. P.; Nerem, R. M. Dynamic mechanical conditioning of collagen-gel blood vessel constructs induces remodeling in vitro. Ann. Biomed. Eng. 28:351-362; 2000.

30. Shin'oka, T.; Imai, Y.; Ikada, Y. Transplantation of a tis- sue-engineered pulmonary artery. N. Engl. J. Med. 344: 532-533; 2001.

31. Shirota, T.; He, H.; Yasui, H.; Matsuda, T. Human endothelial progenitor cell-seeded hybrid graft: Proliferative and antithrombogenic potentials in vitro and fabrication processing. Tissue Eng. 9:127-136; 2003.

32. Simper, D.; Stalboerger, P. G.; Panetta, C. J.; Wang, S.; Caplice, N. M. Smooth muscle progenitor cells in human blood. Circulation 106:1199-11204; 2002.

33. Sodian, R.; Lemke, T.; Fritsche, C.; Hoerstrup, S. P.; Fu, P.; Potapov, E. V.; Hausmann, H.; Hetzer, R. Tissue-engineering bioreactors: A new combined cell-seeding and perfusion system for vascular tissue engineering. Tissue Eng. 8:863-870; 2002.

34. Stegemann, J. P.; Dey, N. B.; Lincoln, T. M.; Nerem, R. M. Genetic modification of smooth muscle cells to control phenotype and function in vascular tissue engineering. Tissue Eng. 10:189-199; 2004.

35. Thompson, C. A.; Colon-Hernandez, P.; Pomerantseva, I.; MacNeil, B. D.; Nasseri, B.; Vacanti, J. P.; Oesterle, S. N. A novel pulsatile, laminar flow bioreactor for the development of tissue-engineered vascular structures. Tissue Eng. 8:1083-1088; 2002.

36. Watanabe, M.; Shin'oka, T.; Tohyama, S.; Hibino, N.; Konuma, T.; Matsumura, G.; Kosaka, Y.; Ishida, T.; Imai, Y.; Yamakawa, M.; Ikada, Y.; Morita, S. Tissue-engineered vascular autograft: Inferior vena cava replacement in a dog model. Tissue Eng. 7:429-439; 2001.

37. Weinberg, C. B.; Bell, E. A blood vessel model constructed from collagen and cultured vascular cells. Science 231:397-400; 1986.

38. Williams, C.; Wick, T. M. Perfusion bioreactor for small diameter tissue-engineered arteries. Tissue Eng. 10:930941; 2004.

39. Zisch, A. H. Tissue engineering of angiogenesis with autologous endothelial progenitor cells. Curr. Opin. Biotechnol. 15:424-429; 2004. 
